\title{
Intoxicação espontânea e experimental por Eupatorium tremulum (Asteraceae) em bovinos ${ }^{1}$
}

\author{
Joelma Lucioli ${ }^{2}$, Fernando Henrique Furlan ${ }^{2}$, Sara Mezaroba ${ }^{3}$, Sandra Davi Traverso ${ }^{4}$ \\ e Aldo Gava ${ }^{4 *}$
}

\begin{abstract}
Lucioli J., Furlan F.H., Mezaroba S., Traverso S. D. \& Gava A. 2007. [Spontaneous and experimental poisoning by Eupatorium tremulum (Asteraceae) in cattle.] Intoxicação espontânea e experimental por Eupatorium tremulum (Asteraceae) em bovinos. Pesquisa Veterinária Brasileira 27(10):442-445. Departamento de Clínica e Patologia, Centro de Ciências Agroveterinárias, Univer-sidade do Estado de Santa Catarina, Lages, SC 88520-000, Brazil. E-mail: a2ag@cav.udesc.br

The spontaneous and experimental poisoning by Eupatorium tremulum in cattle is described. Spontaneous cases were diagnosed in a herd of 19 cattle in the municipality of Lages, Santa Catarina, Brazil. Three of the animals were found dead after having been transferred to a pasture with abundant quantities of $E$. tremulum. On two of them postmortem examination was performed and several internal organs were sampled for histological examination. Green leaves of $E$. tremulum were force-fed orally to 5 calves in single doses of $23-32 \mathrm{~g} / \mathrm{kg}$ body weight. Three calves showed clinical signs and two died. The main clinical signs included anorexia, apathy, absence of rumen movements, diarrhea and a flabby abdominal wall. Gross changes were restricted to the fore stomachs and were identical to those observed in the cases of natural poisoning. Rumen and reticulum were slightly reddish from outside; the corneal layer of their internal lining was loosely attached to a markedly red mucosa. The histological examination of rumen and reticulum from spontaneous and experimental cases revealed necrosis and vesicle formation in the epithelium; in some segments of the ruminal mucosa there was detachment of the epithelial covering and infiltration by neuthophils. Poisoning by E. tremulum has clinical course, gross lesions and histopathology very similar to those observed in poisoning caused by ingestion of the plants Baccharidastrum triplinervium, Baccharis coridifolia and Baccharis megapotamica var. weirii. The diagnosis of the spontaneous cases here described was confirmed by epidemiological data and the experimental reproduction of characteristic gross lesions and histopathology.
\end{abstract}

INDEX TERMS: Poisonous plants, plant poisoning, Eupatorium tremulum, Asteraceae, diseases of cattle, digestive tract.

RESUMO.- Descreve-se a intoxicação natural e experimental por Eupatorium tremulum em bovinos. Um surto de intoxicação espontânea por esta planta foi diagnosticado no município de Lages, Santa Catarina. Em um lote de 19 bovinos, três morreram

\footnotetext{
${ }^{1}$ Recebido em 20 de setembro de 2007.

Aceito para publicação em 5 de outubro de 2007.

${ }^{2}$ Programa de Pós-Graduação em Ciências Veterinárias, área de concentração em Patologia Veterinária, Centro de Ciências Agroveterinárias (CAV), Universidade do Estado de Santa Catarina (UDESC), Av. Luiz de Camões 2090, Bairro Conta Dinheiro, Lages SC 88520-000, Brasil.

3 Graduanda, Curso de Medicina Veterinária, CAV-UDESC, Lages, SC.

${ }^{4}$ Laboratório de Patologia Animal, Departamento de Clínica e Patologia, CAV-UDESC, Lages, SC. *Autor para correspondência: a2ag@cav.udesc.br
}

após a transferência para uma invernada aonde havia grande quantidade de E. tremulum. Os animais foram encontrados mortos, e dois foram necropsiados e coletado amostras de vísceras para exame histológico. Experimentalmente, folhas verdes de $E$. tremulum foram administradas a cinco bovinos, em doses únicas de 23 a $32 \mathrm{~g} / \mathrm{kg}$ de peso animal. Destes, três adoeceram e dois morreram. Os principais sinais clínicos observados foram anorexia, apatia, atonia ruminal, miç̧ão freqüiente e em pequenos jatos, fezes pastosas e ventre flácido. As lesões macroscópicas restringiram-se aos pré-estômagos e foram idênticas, tanto para intoxicação natural como para a experimental. O rúmen e retículo externamente mostravam tonalidade levemente avermelhada, a camada córnea da mucosa estava frouxamente aderida e a mucosa tinha coloração vermelha acentuada. Ao exame 
histológico observou-se no rúmen e retículo, tanto na intoxicação espontânea, como experimental, necrose do epitélio da mucosa com formações de pequenas vesículas e em alguns segmentos, desprendimento da camada epitelial e leve infiltrado de neutrófilos. A intoxicação por $E$. tremulum tem curso clínico, lesões macro e microscópicas muito semelhantes àquelas produzidas pela intoxicação por Baccharidastrum triplinervium, Baccharis coridifolia (mio-mio) e Baccharis megapotamica var. weirii (mio-mio do banhado). O diagnóstico diferencial entre essas quatro intoxicações deve ser feito pela presença da planta e pelos dados epidemiológicos. O diagnóstico dos casos espontâneos foram confirmados pelos dados epidemiológicos e reprodução experimental das lesões macro e microscópicas.

TERMOS DE INDEXAÇÃO: Plantas tóxicas, intoxicação por plantas, Eupatorium tremulum, Asteraceae, intoxicação por planta, doenças de bovinos, sistema digestivo.

\section{INTRODUÇÃO}

O estudo de plantas tóxicas para animais domésticos tem recebido especial atenção devido às perdas econômicas provocadas. $\mathrm{O}$ impacto econômico das intoxicações por plantas é difícil de ser estimado, pois inclui perdas por morte de animais, perda por diminuição de ganho de peso, falhas reprodutivas, diminuição da resposta imunitária, custos com tratamentos, medidas profiláticas e de controle e perdas pela desvalorização dos campos (Riet-Correa \& Méndez 1993).

Na região Sul do Brasil, são freqüientes as mortes de animais causadas pela ingestão de plantas que causam lesões sobre o tubo digestivo. Baccharis coridifolia é a principal planta responsável por causar mortes em bovinos (Tokarnia \& Döbereiner 1975, Barros 1993, Varaschin et al. 1998, Rissi et al. 2005), ovinos (Tokarnia \& Döbereiner 1976, Barros 1993, Rozza et al. 2006) e eqüinos (Costa et al. 1995); Baccharis megapotamica var. weirii (Tokarnia et al. 1992) e Baccharidastrum triplinervium (Langohr et al. 2005) também são responsabilizadas por mortes espontâneas em bovinos.

Na região do Planalto Catarinense com certa frequiência ocorrem mortes de bovinos com quadro clínico e lesões compatíveis com intoxicação por plantas de ação sobre o tubo digestivo. Porém, em alguns surtos, não foi possível relacionar essas mortes com plantas dos gêneros Baccharis e Baccharidastrum. Um desses surtos ocorreu no município de Lages, SC, onde três bovinos morreram. Após avaliação dos dados epidemiológicos e de observação do local, foi constatada a presença de uma planta, conhecida como vassoura do banhado, (posteriormente identificada como Eupatorium tremulum) a qual tinha sido consumida pelos bovinos. Como não foram encontradas outras plantas produtoras de lesões sobre o tubo digestivo, optou-se pela experimentação com essa planta.

O objetivo desse trabalho é verificar a toxicidade de $E$. tremulum para bovinos, conhecer os dados epidemiológicos, a dose letal, os sinais clínicos e as lesões decorrentes da intoxicação por essa planta.

\section{MATERIAL E MÉTODOS}

Um surto de mortalidade de bovinos foi avaliado em uma propriedade do município de Lages, SC, onde um lote de 19 animais, oriundos de outra região, foi introduzido em um piquete. Sendo que 3 dias após a entrada dos animais nessa área, três bovinos foram encontrados mortos.

Folhas verdes de Eupatorium tremulum (Fig.1) foram coletadas e administradas a cinco bovinos em diferentes quantidades. A dose inicial utilizada foi baseada na dose letal de $20 \mathrm{~g} / \mathrm{kg}$, observada na intoxicação experimental por Baccharidastrum triplinervium (Langohr et. al. 2005). As informações sobre quantidade da planta fornecida, número e peso dos animais constam no Quadro 1 . Todos os animais em experimentação foram mantidos fechados em baia, alimentados com capim quicuio (Pennisetum clandestinum), azevém (Lolium multiflorum) e água ad libitum.

Dois animais que morreram espontaneamente e dois que morreram experimentalmente foram necropsiados e, destes foram coletadas amostras de coração, pulmão, rúmen, omaso, abomaso,

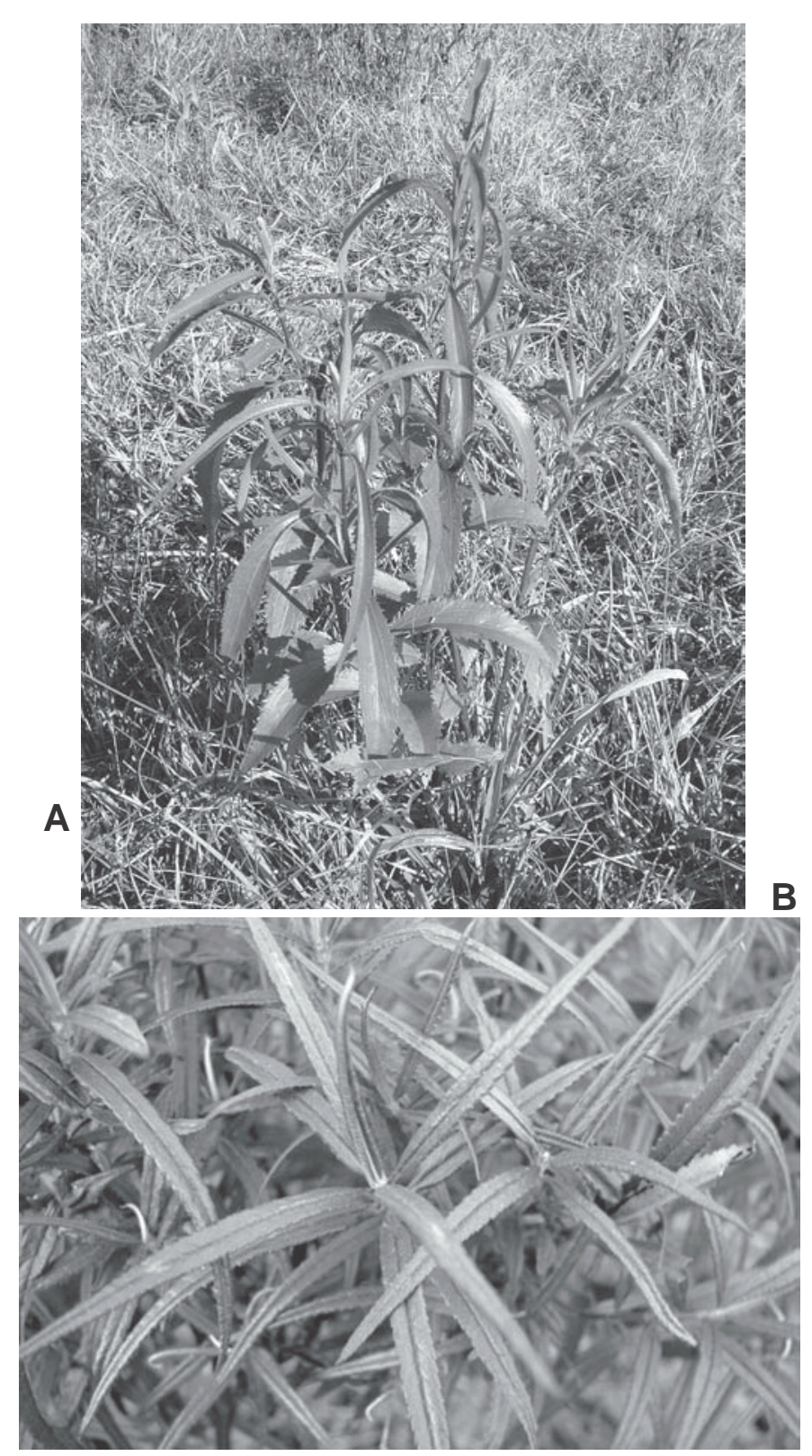

Fig.1. Eupatorium tremulum, município de Lages, SC. (A) Planta jovem, (B) Detalhe da planta adulta. 
Quadro 1. Intoxicação experimental por Eupatorium tremulum em bovinos

\begin{tabular}{|c|c|c|c|c|c|}
\hline $\begin{array}{l}\text { Bovino } \\
\text { no. }^{\mathrm{a}}\end{array}$ & $\begin{array}{l}\text { Dose } \\
(\mathrm{g} / \mathrm{kg})\end{array}$ & $\begin{array}{r}\text { Peso } \\
(\mathrm{kg})\end{array}$ & $\begin{array}{l}\text { Início dos } \\
\text { inais clínicos } \\
\text { após a admi- } \\
\text { nistração da } \\
\text { planta }\end{array}$ & Principais sinais clínicos & $\begin{array}{c}\text { Tempo } \\
\text { decorri- } \\
\text { do até a } \\
\text { morte }\end{array}$ \\
\hline 104 & 30 & 130 & 8 horas & $\begin{array}{l}\text { Anorexia, apatia, movimentos } \\
\text { ruminais diminuídos, micção } \\
\text { em jatos frequente e fezes pas- } \\
\text { tosas, ventre flácido e decúbi- } \\
\text { to lateral }\end{array}$ & 24 horas \\
\hline 109 & 32 & 220 & 20 horas & $\begin{array}{l}\text { Apatia e movimentos ruminais } \\
\text { diminuídos }\end{array}$ & - \\
\hline 115 & 27 & 182 & 16 horas & $\begin{array}{l}\text { Micção em jatos e frequente e } \\
\text { fezes pastosas }\end{array}$ & - \\
\hline 116 & 23 & 99 & 18 horas & $\begin{array}{l}\text { Micção em jatos e frequente e } \\
\text { fezes pastosas }\end{array}$ & - \\
\hline 117 & 30 & 157 & 12 horas & $\begin{array}{l}\text { Anorexia, apatia, movimentos } \\
\text { ruminais diminuídos, micção } \\
\text { em jatos e frequente, fezes } \\
\text { pastosas, ventre flácido e de- } \\
\text { cúbito lateral }\end{array}$ & 27 horas \\
\hline
\end{tabular}

a Número de registro dos animais no Laboratório de Patologia Animal, CAV, UDESC.

intestino, fígado, rim, baço linfonodos, músculo estriado e sistema nervoso central, que foram fixadas em formol a $10 \%$, processadas e coradas pela hematoxilina \& eosina para avaliação histológica.

\section{RESULTADOS}

Dois animais suspeitos de intoxicação espontânea por Eupatorium tremulum foram necropsiados observando-se leve coloração avermelhada da serosa do rúmen e retículo, e coloração vermelha intensa da mucosa dos mesmos. Através da microscopia, foi observado congestão e necrose da mucosa do rúmen e retículo.

Experimentalmente, os Bovinos 104 e 117 que receberam a dose de $30 \mathrm{~g} / \mathrm{kg}$ de E. tremulum morreram 24 e 27 horas, respectivamente, após a administração da planta. O Bovino 109 , que recebeu a planta na dose de $32 \mathrm{~g} / \mathrm{kg}$ adoeceu, mas recuperou-se totalmente após 3 horas do início dos sinais clínicos. Os Bovinos 115 e 116 adoeceram levemente.

Os sinais clínicos iniciaram entre 8 e 20 horas após a administração da planta e foram principalmente: anorexia, apatia, diminuição dos movimentos ruminais, miç̧ão freqüente e em pequenos jatos, fezes pastosas e ventre flácido (Quadro 1).

Na necropsia as lesões se restringiram ao rúmen e retículo, os quais, externamente manifestavam flacidez acentuada e coloração levemente avermelhada da serosa. Internamente o conteúdo era pastoso a liquido, a camada córnea da mucosa desprendia-se facilmente e a mucosa tinha coloração vermelha sendo mais intensa no saco ventral do rúmen e no reticulo.

No exame histológico observou-se no rúmen, necrose do epitélio da mucosa, e infiltrado de neutrófilos de intensidade leve, intercalada por pequenas vesículas ou de vesículas maiores, que culminavam em áreas de desprendimento da camada epitelial (Fig.2.). Além dessas lesões, observou-se ainda congestão dos capilares da mucosa, às vezes acompanhada

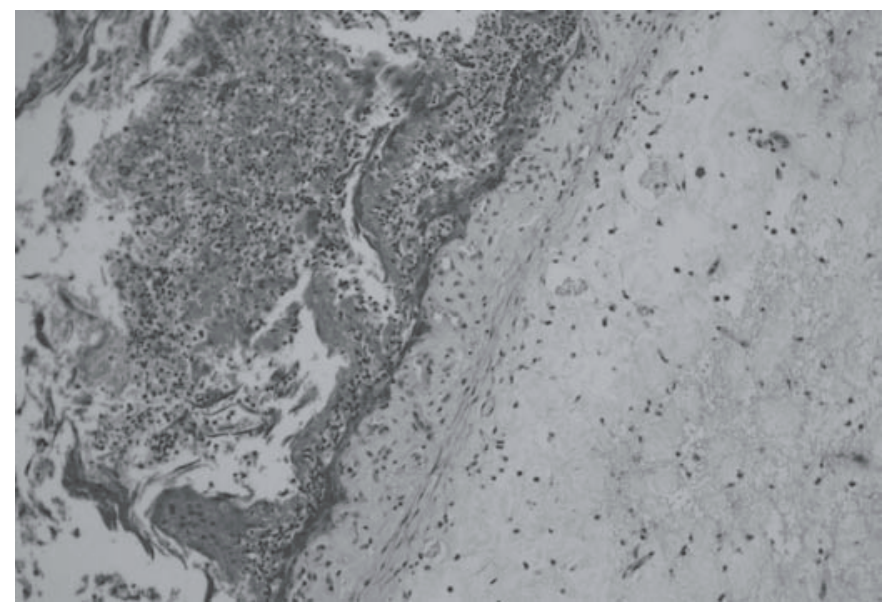

Fig.2. Necrose da camada epitelial e leve infiltrado de neutrófilos, associada a edema de submucosa do rúmen, na intoxicação experimental por Eupatorium tremulum (Bovino 117). HE, obj.10x.

de hemorragia. Nos centros germinativos do baço e linfonodos foi observado eosinofilia e fragmentação celular de intensidade leve a moderada.

\section{DISCUSSÃO}

Eupatorium tremulum mostrou ser tóxico para bovinos a partir de $30 \mathrm{~g} / \mathrm{kg}$, produzindo distúrbios e lesões, principalmente, sobre o tubo digestivo e menos intensamente no tecido linfóide. Desprendimento da camada córnea acompanhada de coloração avermelhada da mucosa ruminal foram as principais alterações macroscópicas. Pela microscopia foi observado vacuolização, necrose e congestão da mucosa do rúmem e retículo. Estas lesões são similares às descritas para outras plantas de ação sobre o tubo digestivo: Baccharis coridifolia (Tokarnia \& Döbereiner 1975, Barros 1993, Varaschin et al. 1998), Baccharis megapotamica var. weirii e var. megapotamica (Tokarnia et al. 1992) e Baccharidastrum triplinervium (Langhor et al. 2005).

Esta similaridade entre as lesões pode dificultar o diagnóstico, tanto a campo, como através da microscopia, uma vez que em muitas áreas do Planalto de Santa Catarina e do Rio Grande do Sul podem ser encontradas na mesma propriedade, $B$. coridifolia, $B$. megapotamica var. weiiri e $B$. triplinervium e E. tremulum. Desta forma, para finalização do diagnóstico é necessária à obtenção de dados epidemiológicos e a verificação do consumo de uma destas plantas pelos bovinos.

A patogenia e a razão exata da causa morte na intoxicação por E. tremulum não foi esclarecida. Porém, devido à semelhança dos sinais clínicos e lesões macro e microscópicas, é provável que sua toxicidade esteja relacionada aos tricotecenos macrociclicos, substâncias essas já isoladas de $B$. coridifolia, B. megapotamica e B. artemisioides (Kupchan et al. 1977, Habermehl et al. 1985, Comezoglu 1987, Jarvis et al. 1987, 1988, 1996, Rizzo et al. 1997).

Os tricotecenos são metabólicos de uma variedade de fungos comuns ao solo (Barros 1993), tendo sido responsabilizados por importantes micotoxicoses ocorridas na Europa, Japão e Estados Unidos em humanos e animais (Armién et al. 
1993). Essas substâncias parecem ser absorvidas e armazenadas por plantas do gênero Baccharis (Habermehl et al. 1985), podendo haver diferenças regionais que afetam a presença dos tricotecenos macrocíclicos (Rizzo et al. 1997).

As condições que favorecem a intoxicação por essas plantas são variáveis. Casos de intoxicação por B. coridifolia ocorrem somente em animais que desconhecem a planta, sendo observados principalmente em bovinos em trânsito e que estão famintos após serem soltos em áreas com a planta (Tokarnia \& Döbereiner 1975). B. megapotamica var. weirii é ingerida quando há escassez de pastos, durante secas pronunciadas, ao passo que a variedade megapotamica parece não ser consumida por bovinos (Tokarnia et al. 1992). A ingestão de E. tremulum, à semelhança das plantas acima citadas também parece estar relacionada aos bovinos que desconhecem a planta e com carência alimentar.

E. tremulum foi letal para bovinos em doses de $30 \mathrm{~g} / \mathrm{kg}$. Desta forma, a toxicidade desta é semelhante à observada $\operatorname{com} B$. triplinervium que foi de $20 \mathrm{~g} / \mathrm{kg}$ (Langhor et al. 2005). Para $B$. coridifolia, as doses tóxicas letais da planta verde são de 0,25 $0,5 \mathrm{~g} / \mathrm{kg}$ na época de floração e de $2 \mathrm{~g} / \mathrm{kg}$ na época de brotação (Tokarnia \& Döbereiner 1975, Varaschin et al. 1998). Para B. megapotamica var. weirii a dose letal em bovinos é de $1 \mathrm{~g} / \mathrm{kg}$ e a var. megapotamica, de $3-4 \mathrm{~g} / \mathrm{kg}$, independentemente do ciclo vegetativo e do sexo das plantas (Tokarnia et al. 1992).

Na intoxicação por plantas que afetam primariamente o sistema digestivo, costumam ser utilizados como tratamento, vários fármacos incluindo protetores de mucosa, protetores hepáticos e glicose, sem que haja experimentos que atestem sua eficácia (Barros 1993). O controle da intoxicação por E. tremulum ainda deve ser estabelecido. Medidas profiláticas que evitem o contato com a planta devem ser recomendados.

O diagnóstico diferencial da intoxicação por essas quatro plantas deve ser feito pela presença da planta consumida no local e dados epidemiológicos.

\section{CONCLUSÕES}

Eupatorium tremulum produz lesões sobre o tubo digestivo, provocando alterações clínicas quando ingerido em dose única a partir de $23 \mathrm{~g} / \mathrm{kg}$, podendo levar a morte quando ingerida em dose igual ou superior a $30 \mathrm{~g} / \mathrm{kg}$.

As lesões macro e microscopicamente encontradas na forma espontânea foram idênticas às produzidas pela intoxicação experimental por E. tremulum.

Essa planta pode ser responsabilizada por mortalidade em bovinos na região sul do Brasil.

Agradecimentos.- Ao Professor Nelson Matzenbacher, da Universidade Federal do Rio Grande do Sul, pela classificação botânica de Eupatorium tremulum.

\section{REFERÊNCIAS}

Armién A.G., Peixoto P.V. \& Tokarnia C.H. 1993. Intoxicação experimental por Baccharis megapotamica var. megapotamica e var. weirii (Compositae) em ovinos. Pesq. Vet. Bras. 13(1/2):5-20.

Barros C.S.L. 1993. Intoxicação por Baccharis coridifolia, p.159-169. In: RietCorrea F., Méndez M.C. \& Schild A.L. (ed.), Intoxicações por Plantas e Micotoxicoses em Animais Domésticos. Agropecuaria Hemisferio Sur S.R.L., Montevideo.

Comezoglu S.N. 1987. Isolation and characterization of new trichothecenes from Baccharis megapotamica; chemical modifications of baccharinoid B5. Diss. Abstr. Int. (Sci.) 47(9):376-378.

Costa E.R., Costa J.N., Armién A.G., Barbosa J.D. \& Peixoto P.V. 1995. Intoxicação experimental por Baccharis coridifolia (Asteraceae) em eqüinos. Pesq. Vet. Bras. 15(1):19-26.

Habermehl G.G., Busam L., Heydel P.L., Mebs D., Tokarnia C.H. Döbereiner J. \& Spraul M. 1985. Macrocyclic trichothecenes: cause of livestock poisoning by the Brazilian plant Baccharis coridifolia. Toxicon 23(5):731-745.

Jarvis B.B., Comezoglu S.N., Ammon H.I., Breedlove C.K., Miller R.W., Woode M.K., Streelman D.R., Sneden A.T., Dailey R.G. Jr \& Kupchan S.M. 1987. New macrocyclic trichothecenes from Baccharis megapotamica. J. Nat. Prod. 50(5):815-828.

Jarvis B.B., Midiwo J.O., Bean G.A., Aboul-Nasr M.B. \& Barros C.S. 1988. The mystery of trichothecene antibiotics in Baccharis species. J. Nat. Prod. 51(4):736-744.

Jarvis B.B., Wang S., Cox C., Rao M.M., Philip V., Varaschin M.S. \& Barros C.S. 1996. Brazilian Baccharis toxins: livestock poisoning and the isolation of macrocyclic trichothecene glucosides. Nat. Toxins 4(2):58-71.

Kupchan S.M., Streelman D.R., Jarvis B.B., Daily R.G. \& Sneden A.T. 1977. Isolations of potent new antileukemic trichothecenes from Baccharis megapotamica. J. Org. Chem. 42(26):4221-4225.

Langohr I.M., Gava A. \& Barros C.S.L. 2005. Intoxicação natural e experimental por Baccharidastrum triplinervuim (Asteraceae) em bovinos. Pesq. Vet. Bras. 25(4):235-238.

Riet-Correa F. \& Méndez M.C. 1993. Introdução ao estudo das plantas tóxicas, p.1-5. In: Riet-Correa F., Méndez M.C. \& Schild A.L. Intoxicação por Plantas e Micotoxicoses em Animais Domésticos. Editora Hemisfério Sul do Brasil, Pelotas.

Rissi D.R., Rech R.R., Fighera R.A., Cagnini D.Q., Kommers G.D. \& Barros C.S.L. 2005. Intoxicação espontânea por Baccharis coridifolia em bovinos. Pesq. Vet. Bras. 25(2):111-114.

Rizzo I.L., Varsavky E., Haidukowski M. \& Frade H. 1997. Macrocyclic trichothecenes in Baccharis coridofolia plants and endophytes and Baccharis artemisioides plants. Toxicon 35(5):753-757.

Rozza D.B., Raymundo D.L., Corrêa A.M.R., Leal J., Seitz A.L., Driemeier D. \& Colodel, E.M. 2006. Intoxicação espontânea por Baccharis coridifolia (Compositae) em ovinos. Pesq. Vet. Bras. 26(1):21-25.

Tokarnia C.H. \& Döbereiner J. 1975. Intoxicação experimental em bovinos por "mio-mio" (Baccharis coridifolia). Pesq. Vet. Bras. 10:79-97.

Tokarnia C.H. \& Döbereiner J. 1976. Intoxicação experimental em ovinos por "mio-mio" (Baccharis coridifolia). Pesq. Vet. Bras. 11:19-26.

Tokarnia C.H., Peixoto P.V., Gava A. \& Barros C.S.L. 1992. Intoxicação experimental por Baccharis megapotamica var. megapotamica e var. weirii (Compositae) em bovinos. Pesq. Vet. Bras. 12(1/2):19-31.

Varaschin M.S., Barros C.S.L. \& Jarvis B.B. 1998. Intoxicação experimental por Baccharis coridifolia (Compositae) em bovinos. Pesq. Vet. Bras. 18(2):69-75. 